\title{
A copper-containing oxytelluride as a promising thermoelectric material for waste heat recovery
}

Article

Accepted Version

Vaqueiro, P., Gouelou, G., Stec, M., Guilmeau, E. and Powell, A. (2013) A copper-containing oxytelluride as a promising thermoelectric material for waste heat recovery. Journal of Materials Chemistry A, 1 (3). pp. 520-523. ISSN 0959-9428 doi: https://doi.org/10.1039/c2ta00878e Available at https://centaur.reading.ac.uk/34242/

It is advisable to refer to the publisher's version if you intend to cite from the work. See Guidance on citing.

Published version at: http://pubs.rsc.org/en/content/articlelanding/2013/ta/c2ta00878e\#!divAbstract

To link to this article DOI: http://dx.doi.org/10.1039/c2ta00878e

Publisher: Royal Society of Chemistry

All outputs in CentAUR are protected by Intellectual Property Rights law, including copyright law. Copyright and IPR is retained by the creators or other copyright holders. Terms and conditions for use of this material are defined in the End User Agreement.

www.reading.ac.uk/centaur 
Central Archive at the University of Reading

Reading's research outputs online 


\title{
A copper-containing oxytelluride as a promising thermoelectric material for waste heat recovery
}

\author{
Paz Vaqueiro, ${ }^{* a}$ Gabin Guelou, ${ }^{a}$ Maria Stec, ${ }^{a}$ Emmanuel Guilmeau ${ }^{b}$ and Anthony V Powell ${ }^{a}$ \\ Received (in $X X X, X X X) X t h X X X X X X X X X 20 X X$, Accepted Xth $X X X X X X X X X 20 X X$ \\ 5 DOI: 10.1039/b000000x
}

The new thermoelectric material BiOCuTe exhibits an electrical conductivity of $224 \mathrm{~S} \mathrm{~cm}^{-1}$ and a Seebeck coefficient of $+186 \mu \mathrm{V} \mathrm{K}^{-1}$ at $373 \mathrm{~K}$, together with an extremely low lattice thermal conductivity of $\sim 0.5 \mathrm{~W} \mathrm{~m}^{-1} \mathrm{~K}^{-1}$. This results in ${ }_{10} \mathrm{a}$ ZT of 0.42 at $373 \mathrm{~K}$, which increases to 0.66 at the maximum temperature investigated, $673 \mathrm{~K}$.

Thermoelectric power generation is a promising technology to 15 convert waste heat into electricity. This has led to a tremendous growth in research into new materials with high thermoelectric performance. This performance is normally expressed in terms of a figure of merit, ZT, related to the Seebeck coefficient $(S)$, electrical conductivity $(\sigma)$ and 20 thermal conductivity $(\kappa)$ of the material by $\mathrm{ZT}=\mathrm{S}^{2} \sigma \mathrm{T} / \kappa{ }^{1}$ Given that vast quantities of low-grade waste heat $(T<623$ $\mathrm{K}$ ) are discharged into the earth's environment every year, it is highly desirable to develop thermoelectric generators suited for these temperatures. ${ }^{2}$ There is however a paucity of 25 candidate thermoelectric materials suitable for operation over the temperature range $273 \leq \mathrm{T} / \mathrm{K} \leq 623 .^{3}$ Currently, power generation at near ambient temperatures is carried out using heavily-doped $\mathrm{Bi}_{2} \mathrm{Te}_{3}$, with a maximum value of $\mathrm{ZT} \approx 1$ at $380 \mathrm{~K}$.

$30 \mathrm{~A}$ promising approach to improve thermoelectric performance is to design materials containing "natural superlattices", 4 in which layers with excellent electronic transport properties are combined with a second type of layer which serves as a phonon scatterer. Evidence for the success of this approach 35 can be found in the outstanding thermoelectric properties of layered cobalt oxides, ${ }^{5}$ the misfit layered compounds $(\mathrm{SnS})_{1+\mathrm{x}}\left(\mathrm{TiS}_{2}\right)(\mathrm{ZT}=0.37$ at $773 \mathrm{~K}), 4$ and the oxyselenide $\mathrm{BiOCu}_{1-\mathrm{x}} \mathrm{Se}(\mathrm{ZT}=0.81$ at $923 \mathrm{~K}){ }^{6}$ However, whilst these results demonstrate the potential of the "natural superlattice" 40 concept, ZT values over the temperature range required for low-grade waste heat recovery $(\mathrm{T}<623 \mathrm{~K})$ are significantly lower than that of $\mathrm{Bi}_{2} \mathrm{Te}_{3}$. In seeking to identify materials suitable for thermoelectric generation below $623 \mathrm{~K}$, we have taken into account the relationship between maximum $\mathrm{ZT}$ and ${ }_{45}$ band gap, ${ }^{7}$ and thus focused our work on naturally layered phases with band gaps close to those of $\mathrm{Bi}_{2} \mathrm{Te}_{3}(0.13 \mathrm{eV})^{8}$ and PbTe $(0.33 \mathrm{eV}) .7$ Within the family of oxychalcogenides AOBQ $(\mathrm{A}=\mathrm{La}, \mathrm{Ce}, \mathrm{Nd}, \mathrm{Pr}, \mathrm{Bi} ; \mathrm{B}=\mathrm{Cu}, \mathrm{Ag}$ and $\mathrm{Q}=\mathrm{S}, \mathrm{Se}$, $\mathrm{Te})^{9}$ bismuth-containing phases are particularly interesting,
50 because they have significantly lower band gaps than those containing rare earths, due to the contribution of $\mathrm{Bi} 6 p$ states to the bottom of the conduction band. ${ }^{10}$ The lowest band gap, estimated as $0.2-0.5 \mathrm{eV}$, occurs for BiOCuTe. $9^{(\mathrm{e})}$ This led us to study, for the first time, the thermoelectric properties of ${ }_{55} \mathrm{BiOCuTe}$. We also investigated the effect of $\mathrm{Pb}^{2+}$ as a dopant on ZT. We demonstrate here that, in accord with our reasoning above, BiOCuTe has a remarkable thermoelectric performance at temperatures suitable for waste heat recovery, with values of $\mathrm{ZT}$ of $0.42-0.60$ over the temperature range 373 ${ }_{60} \leq \mathrm{T} / \mathrm{K} \leq 623$. For BiOCuTe, $\mathrm{ZT}$ at $673 \mathrm{~K}(\mathrm{ZT}=0.66)$ is significantly higher than that for $\mathrm{BiOCu}_{1-\mathrm{x}} \mathrm{Se}(\mathrm{ZT} \sim 0.3) 6$ or $(\mathrm{SnS})_{1+\mathrm{x}}\left(\mathrm{TiS}_{2}\right)(\mathrm{ZT} \sim 0.32) 4$ at the same temperature.
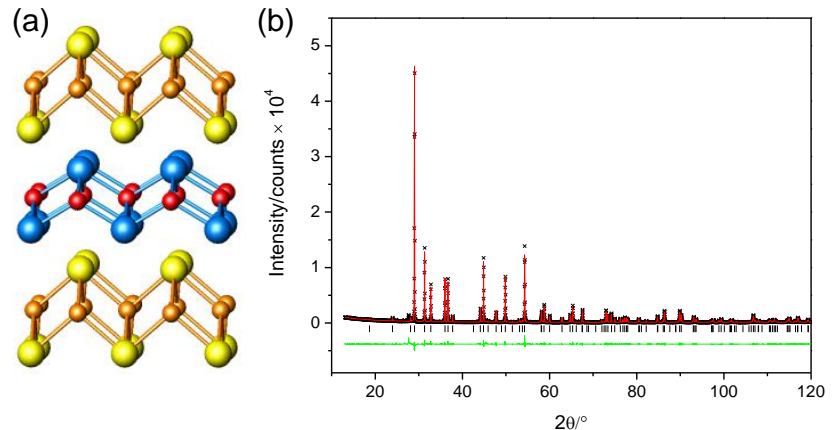

Figure 1. (a) View of the crystal structure of BiOCuTe along the [010] 65 direction. Key: copper, orange; tellurium, yellow; bismuth, blue; oxygen, red; (b) Rietveld refinement using powder X-ray diffraction data for $\operatorname{BiOCuTe}\left(\mathrm{R}_{\mathrm{wp}}=12.4 \%\right)$.

Samples with composition $\mathrm{Bi}_{1-\mathrm{x}} \mathrm{Pb}_{\mathrm{x}} \mathrm{OCuTe}(0 \leq \mathrm{x} \leq 0.08)$ were prepared by solid-state synthesis at temperatures in the 70 range $623 \leq \mathrm{T} / \mathrm{K} \leq 773$. For transport measurements, the asprepared powders were hot pressed into well-densified pellets (Supporting Information). Analysis of powder X-ray diffraction data indicates that BiOCuTe and all doped samples crystallize in the $\mathrm{ZrSiCuAs}$ structure (space group P4/nmm), 75 which can be described as composed of alternating fluoriteand antifluorite-type layers, stacked along [001] (Figure 1(a)). These layers consist of edge-sharing $\mathrm{OBi}_{4}$ and $\mathrm{CuTe}_{4}$ tetrahedra respectively. Rietveld refinements (Figure 1(b) and Supplementary Information) indicate that the $a$ axis decreases 80 slightly with increasing lead content, whilst there is a relatively large expansion of the unit cell along the $c$ axis. This results in an increase of the Bi-Te distances, which may 
indicate a weakening of the bonding between the oxide and chalcogenide layers with increasing lead content. The thermal parameter of the copper site is significantly larger than those of the other crystallographic sites. Attempts to refine the 5 occupancy of the copper site led to values below 1, but with large standard deviations. This may be indicative of copper deficiency, an observation which would be consistent with previous work on the related phases MCuFQ $(\mathrm{M}=\mathrm{Sr}, \mathrm{Eu})$, which show copper deficiency. ${ }^{11}$ Thermogravimetric analysis 10 of BiOCuTe shows that, under an inert $\mathrm{N}_{2}$ atmosphere, this material is thermally stable up to at least $873 \mathrm{~K}$. By contrast, under a pure $\mathrm{O}_{2}$ atmosphere, the onset of oxidation occurs at $543 \mathrm{~K}$, a temperature comparable to those at which some high-temperature thermoelectric materials such as 15 skutterudites oxidize (e.g. $\mathrm{CeFe}_{4} \mathrm{Sb}_{12}$ begins to oxidize at 573 $\mathrm{K}) .{ }^{12}$

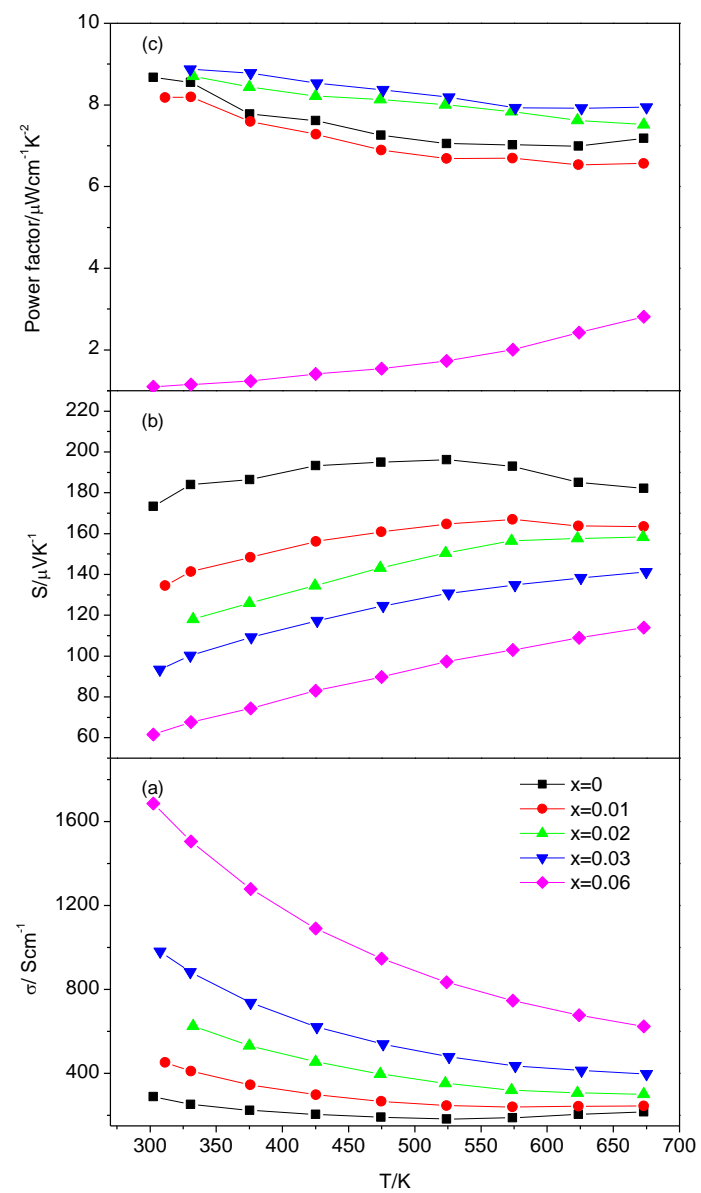

Figure 2. Temperature dependence, over the temperature range $298<\mathrm{T} /$ $\mathrm{K} \leq 673$, of the (a) electrical conductivity, (b) Seebeck coefficient and (c) 20 power factor for $\mathrm{Bi}_{1-x} \mathrm{~Pb}_{\mathrm{x}} \mathrm{OCuTe}$.

The electrical conductivity of as-prepared BiOCuTe has a reasonably high value of $289 \mathrm{Scm}^{-1}$ at $300 \mathrm{~K}$. The conductivity decreases with increasing temperature (Figure 2(a) and Supplementary Information), reaching a minimum 25 value of $181 \mathrm{Scm}^{-1}$ at $523 \mathrm{~K}$. The Seebeck coefficient exhibits an almost linear temperature dependence up to $523 \mathrm{~K}$, and reaches a maximum value of $196 \mu \mathrm{VK}^{-1}$ at this temperature (Figure 2(b)). The Seebeck coefficient indicates that the majority charge carriers in BiOCuTe are holes, and is
30 consistent with p-type semiconducting behaviour. The origin of this p-type behaviour may be related to copper deficiency, which generates mobile holes and leads to the p-type conduction normally observed for this family of oxychalcogenides. 9 The metal-like temperature dependence of 35 the electrical conductivity may be related to copper defficiency or to a a shallower valence band maximum. $9^{(\mathrm{e})}$ The temperature dependence of the electrical conductivity and the Seebeck coefficient up to $523 \mathrm{~K}$ are characteristic of conduction by extrinsic charge carriers, and indicate that ${ }_{40} \mathrm{BiOCuTe}$ is a degenerate semiconductor. The reduction in the Seebeck coefficient above $523 \mathrm{~K}$ can be attributed to the increase in minority charge carriers which occurs in the intrinsic region. The band gap of $\mathrm{BiOCuTe}$ can be estimated from the maximum value of the Seebeck coefficient $\left(S_{\max }\right)$, 45 using the expression, $\mathrm{S}_{\max }=\mathrm{E}_{\mathrm{g}} /\left(2 e \mathrm{~T}_{\max }\right)$ where $\mathrm{E}_{\mathrm{g}}$ is the band gap energy, $e$ is the electron charge and $\mathrm{T}_{\max }$ is the absolute temperature at which the maximum occurs. ${ }^{13}$ This leads to an estimated value of $0.21 \mathrm{eV}$ for the band gap of BiOCuTe, which is in reasonable agreement with those previously 50 determined by optical measurements $(0.4-0.5 \mathrm{eV})$ and DFT calculations $(0.2 \mathrm{eV}) .9^{(\mathrm{e})}$ Data on doped samples (Figure 2 and Supplementary Information) indicate that lead doping results in a progressive increase of the electrical conductivity, and a simultaneous reduction of the Seebeck coefficient. This 55 results in a small enhancement of the power factor for samples with a lead content $\mathrm{x} \leq 0.03$ (Figure 2(c)).

The thermal conductivity of BiOCuTe (Figure 3(a)) is remarkably low when compared to $\mathrm{Bi}_{2} \mathrm{Te}_{3}\left(\sim 2 \mathrm{~W} \mathrm{~m}^{-1} \mathrm{~K}^{-1}\right) .8$ The electronic and lattice contributions were estimated using the 60 electrical conductivity data in conjunction with the Wiedemann-Franz law, with a Lorenz constant of $2.45 \times 10^{-8} \mathrm{~W} \Omega$ $\mathrm{K}^{-2}$. This results in a lattice thermal conductivity of only 0.47 $\mathrm{W} \mathrm{m}{ }^{-1} \mathrm{~K}^{-1}$ for BiOCuTe at $373 \mathrm{~K}$, indicating that the phonon mean free path in this material is rather small. The total ther-

65 mal conductivity increases with increasing lead content, because the electronic contribution rises with $\mathrm{x}$, whilst the lattice thermal conductivity remains almost constant. For BiOCuTe, the electronic thermal conductivity is ca. $30 \%$ of the total, while at the maximum doping level investigated $(x=0.08)$, 70 the electronic thermal conductivity reaches $c a .65 \%$ of the total. The low thermal conductivity of BiOCuTe may arise from the two-dimensional nature of the structure of this material. Band structure calculations on AOBQ phases indicate that the bonding is highly anisotropic, ${ }^{14}$ with the 75 charge carriers confined in the $\left[\mathrm{B}_{2} \mathrm{Q}_{2}\right]$ layers, and that the nature of the bonding between the $\left[\mathrm{A}_{2} \mathrm{O}_{2}\right]$ and $\left[\mathrm{B}_{2} \mathrm{Q}_{2}\right]$ layers is mainly electrostatic and weaker than the intralayer bonding. ${ }^{15}$ Scattering of phonons at the interfaces between these layers may be the origin of the low thermal conductivity. 4 In 80 addition, we can draw some parallels between BiOCuTe and the recently discovered thermoelectric material $\mathrm{Cu}_{2-\mathrm{x}} \mathrm{Se},{ }^{16}$ that may explain the low thermal conductivity of the former. $\mathrm{Cu}_{2-}$ ${ }_{x} \mathrm{Se}$, a superionic conductor with the antifluorite structure, has an extremely low thermal conductivity, attributed to the 85 presence of a highly disordered copper ion sublattice. In the AOBQ family to which BiOCuTe belongs, ionic mobility of the transition metal ions within the antifluorite $\left[\mathrm{B}_{2} \mathrm{Q}_{2}\right]$ layers 
has also been reported. ${ }^{17}$ Fast-ion conduction has been shown in $\mathrm{LaOAgS},{ }^{17(\mathrm{~b})}$ whilst copper can be readily extracted and inserted from $\mathrm{CeOCuS}$ under mild conditions. ${ }^{17(\mathrm{c})}$ By contrast, the ionic conductivity of $\mathrm{BiOCuSe}$ is reported to be 5 negligible. ${ }^{18}$ To the best of our knowledge the ionic conductivity of BiOCuTe has not been investigated, but the large magnitude of copper thermal parameter arising from Rietveld refinements suggest copper deficiency, disorder in the copper sublattice and/or mobility. This may be a 10 contributory factor to the low thermal conductivity of this material, and should be investigated further, as ionic conductivity may cause degradation of the thermoelectric device. ${ }^{19}$

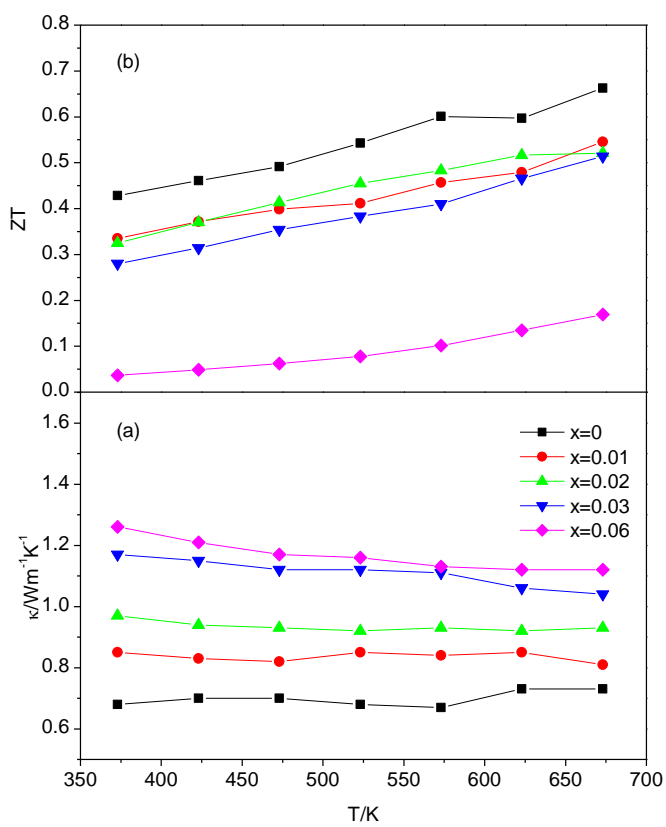

15 Figure 3. Temperature dependence of the (a) thermal conductivity and (b) $\mathrm{ZT}$, for $\mathrm{Bi}_{1-\mathrm{x}} \mathrm{Pb}_{\mathrm{x}} \mathrm{OCuTe}$.

The power factor of BiOCuTe $\left(8.7 \mu \mathrm{W} \mathrm{cm}{ }^{-1} \mathrm{~K}^{-2}\right.$ at $\left.298 \mathrm{~K}\right)$ is moderate in comparison to that of $\mathrm{Bi}_{2} \mathrm{Te}_{3}\left(\sim 40 \mu \mathrm{W} \mathrm{cm}{ }^{-1} \mathrm{~K}^{-}\right.$ $\left.{ }^{2}\right), 8$ but similar to those of other thermoelectric materials such 20 as $\mathrm{Zn}_{4} \mathrm{Sb}_{3}\left(\sim 13 \mu \mathrm{W} \mathrm{cm}{ }^{-1} \mathrm{~K}^{-2} \text { at } 673 \mathrm{~K}\right)^{20}$ or $\mathrm{BiOCu}_{1-\mathrm{x}} \mathrm{Se}(\sim 4$ $\mu \mathrm{W} \mathrm{cm} \mathrm{K}^{-1}$ at $\left.923 \mathrm{~K}\right) .6$ Because of its low thermal conductivity, BiOCuTe exhibits remarkable values of the thermoelectric figure of merit, ZT, (Figure 3(b)) at temperatures suitable for waste heat recovery. As ZT 25 continues to increase with increasing temperature, this material may exhibit even higher ZT values at higher temperatures. Our preliminary work on doping indicates that, although low levels of lead doping result in an enhancement of the power factor, $\mathrm{ZT}$ is reduced due to the simultaneous 30 increase in electronic thermal conductivity. This suggests that further efforts on BiOCuTe are required to optimise the charge carrier concentration, and in particular, to investigate samples with smaller charge carrier concentrations. Given the promising thermoelectric performance found for as-prepared ${ }_{35} \mathrm{BiOCuTe}$, we believe that there is considerable scope for the improvement of ZT through careful control of the stoichiometry and doping. The outstanding thermoelectric properties of $\mathrm{BiOCu}_{1-\mathrm{x}} \mathrm{Se} 6$ and those reported here for
BiOCuTe suggest that the antifluorite $\left[\mathrm{Cu}_{2} \mathrm{Q}_{2}\right]^{2-}$ slabs have 40 remarkable electronic and thermal transport properties. This opens up a new direction for research in thermoelectrics, as other phases containing $\left[\mathrm{Cu}_{2} \mathrm{Q}_{2}\right]^{2-}$ slabs should also be screened as prospective thermoelectric materials.

\section{${ }_{45}$ Notes and references}

${ }^{a}$ Institute of Chemical Sciences \& Centre for Advanced Energy Storage and Recovery (CAESAR), Heriot-Watt University, Edinburgh EH14 4AS, UKE-mail: P.Vaqueiro-Rodriguez@hw.ac.uk

${ }^{b}$ Laboratoire CRISMAT, UMR 6508 CNRS/ENSICAEN, 6 bd. du 50 Maréchal Juin, F-14050 CAEN Cedex 4-France

$\dagger$ Electronic Supplementary Information (ESI) available: Experimental details, powder X-ray diffraction data and Rietveld refinements, lowtemperature electrical conductivity and Seebeck coefficient, thermogravimetric analysis, lattice and electronic thermal conductivity 55 data. See DOI: 10.1039/b000000x/

1 D.M. Rowe, Thermoelectrics Handbook: Macro to Nano, Ed. D.M Rowe, CRC Press, Boca Raton, FL, 2006, Chapter 1.

2 T. Kajikawa, Thermoelectrics Handbook: Macro to Nano, Ed. D.M. Rowe, CRC Press, Boca Raton, FL, 2006, Chapter 50.

3 J.R. Sootsman, D.Y. Chung, M.G. Kanatzidis, Angew. Chem.Int. Ed. 2009, 48, 8616.

4 C. Wan, Y. Wang, N. Wang, W. Norimatsu, M. Kusunoki, K. Koumoto, Sci. Technol. Adv. Mater., 2010, 11, 044306.

5 I. Terasaki, Y. Sasago, K. Uchinokura, Phys. Rev. B 1997, 56, R12685.

6 Y. Liu, L.-D. Zhao, Y. Liu, J. Lan, X. Wei, F. Li, B.P. Zhang, D. Berardan, N. Dragoe, Y.-H. Lin, C.-W. Nan, J.-F. Li, H. Zhu, J. Am. Chem. Soc. 2011, 133, 20112.

7 C. Wood, Rep. Prog. Phys. 1988, 51, 459.

8 H. Scherrer, S. Scherrer CRC Handbook of Thermoelectrics, Ed. D.M. Rowe, CRC Press, Boca Raton, FL, 1995, Chapter 19.

9 (a) M. Palazzi, C. Carcaly, J. Flahaut, J. Solid State Chem. 1980, 35, 150; (b) A.M. Kusainova, P.S. Berdonosov, L.G. Akselrud, L.N. Kholodkovskaya, V.A. Dolgikh, B.A. Popovkin, J. Solid State Chem. 1994, 112, 189; (c) G.H. Chan, B.Den, M.Bertoni, J.R. Ireland, M.C. Hersam, T.O. Mason, R.P. Van Duyne, J.A. Ibers, Inorg. Chem. 2006, 45, 8264; (d) M.L. Liu, L.B. Wu, F.Q. Huang, L.D. Chen, J.A. Ibers, J. Solid State Chem. 2007, 180, 62; (e) H. Hiramatsu,H. Yanagi, T.Kamiya, K. Ueda, M. Hirano, H. Hosono, Chem. Mater. 2008, 20, 326.

10 S. Sallis, L.F.J. Piper., J. Francis, J. Tate, H. Hiramatsu, T. Kamiya, H. Hosono, Phys. Rev. B 2012, 85, 085207.

11 E. Motomitsu, H. Yanagi, T. Kamiya, M. Hirano, H. Hosono, J. Solid State Chem. 2006, 179, 1668.

12 A.C. Sklad, M.W. Gaultois, A.P. Grosvenor, J. Alloys Comp. 2010, 505, L6.

13 H.J. Goldsmid, J.W. Sharp, J. Electron. Mater. 1999, 28, 869.

14 K. Ueda, H. Hiramatsu, H. Ohta, M. Hirano, T. Kamiya, H. Hosono, Phys. Rev. B, 2004, 69, 155305.

15 V.V. Bannikov, I.R. Shein, A.L. Ivanovskii, Solid State Sci. 2012, 14, 89.

16 H. Liu, X. Shi, F. Xu, W. Zhang, L. Chen, Q. Li, C. Uher, T. Day, G.J. Snyder, Nature Mater. 2012, 11, 422.

17 (a) S.J. Clarke, P. Adamson, S.J.C. Herkelrath, O.J. Rutt, D.R. Parker, M.J. Pitcher, C.F. Smura, Inorg. Chem. 2008, 47, 8473; (b) D. Wilmer, J.D. Jorgensen, B.J. Wuensch, Solid State Ionics 2000, 136-137, 961; (c) M.J. Pitcher, C.F. Smura, S.J. Clarke, Inorg. Chem. 2006, 48, 9054.

18 C. Barreteau, D. Berardan, E. Amzallag, L. Zhao, N. Dragoe, Chem. Mater., 2012, 24, 3168

19 F. Gascoin, A. Maignan, Chem. Mater., 2011, 23, 2510.

20 T. Caillat, J.-P. Fleurial, A. Borshchevsky, J. Phys. Chem. Solids 1997, 58, 1119. 